\title{
EDITORIAL:
}

\section{RESIDUOS MINEROS, UNA FUENTE ALTERNATIVA DE MATERIAS PRIMAS}

\author{
Edward Restrepo $^{1 *}$, Erwin Ciro $^{2}$ \\ 1: Ph.D., Catedrático Universidad de Antioquia, GIPIMME-GIMACYR, Medellín, Colombia \\ 2:Ph.D., Candidate, Department Chemical Engineering Materials Environment, Sapienza University of \\ Rome, Via Eudossiana 18, I-00184 Roma, Italy. \\ *edward.restrepo@udea.edu.co
}

Uno de los pilares fundamentales de la producción de bienes, servicios y de infraestructura, es la minería. Esta actividad genera cantidades significativas de residuos, que pueden ser utilizados como una fuente potencial de recursos mediante su reincorporación en la línea productiva de diferentes tipos de industrias. De esta manera, el máximo beneficio tanto de los minerales extraídos como de sus respectivos residuos juega un papel significativo en la minería actual, que busca no solo la optimización de sus procesos, sino también preservar el medio ambiente.

Solo en 2019, el mercado global de residuos mineros fue de aproximadamente $196 \mathrm{mil}$ millones de toneladas y se estima que alcanzará casi 290 mil millones de toneladas en 2026, como consecuencia de la mayor demanda de los minerales comúnmente extraídos por los países en desarrollo [1]. Incluso la generación de estos desechos puede aumentar aún más, debido al continuo crecimiento de las tecnologías de energías limpias, lo que ha llevado a un mayor consumo de minerales como el $\mathrm{Au}, \mathrm{Li}, \mathrm{Co}$ y grafito [2]. Según el Banco Mundial, la producción de grafito, litio y cobalto podría experimentar entre 2021 y 2050, un aumento de aproximadamente $500 \%$, con el fin de satisfacer la demanda de energías verdes. Por lo tanto, se estima que se requerirán más de 3000 millones de toneladas de minerales y metales para la implementación de la energía eólica, solar y geotérmica, así como para el almacenamiento de energía [3].

Las actuales demandas mineras sugieren entonces la necesidad de adoptar nuevas metodologías extractivas mucho más flexibles, dinámicas e innovativas, basadas en la optimización de la línea de extracción, junto a la viabilidad económica en términos de la disminución del consumo energético y de la huella ambiental. Estas exigencias también involucran los residuos mineros, donde su conversión en productos valiosos, es decir reciclaje, es una exigencia desde diferentes perspectivas, para apuntar a la implementación de los principios fundamentales de la economía circular. De esta manera, se requieren propuestas que permitan el desarrollo de una minería amigable con el medio ambiente, siendo factibles y económicamente viables, mientras la mitigación y tratamiento de los residuos producidos se mantiene como una necesidad intrínseca del proceso extractivo.

El presente número de la Revista Colombiana de Materiales presenta 
resultados de importantes trabajos de investigación, donde han sido utilizados residuos provenientes de la minería del oro, desechos generados por la combustión de carbón mineral, empleado como fuente de energía en procesos industriales, y los resultados de la calcinación de rocas fosfóricas, como fuente de materia prima para elaborar productos que podrían ser usados por la industria cerámica, del vidrio y agrícola. Además, se presenta un estudio sobre el drenaje acido de relaves mineros donde se definen comportamientos oxidativos naturales como resultado de los efectos de la intemperie.

Estos trabajos contribuyen a fomentar la transición hacia un mundo más eficiente y sostenible, aprovechando los residuos actualmente disponibles para la producción de nuevas materias primas. Tal producción, podría contribuir a la disminución de extracción de minerales y del consumo energético, como también a minimizar efectos negativos sobre el medio ambiente y la salud de los seres vivos.

\section{REFERENCIAS}

[1] Research and Markets, MINING WASTE MANAGEMENT MARKET, BY MINING METHOD, METALS/MINERALS， BY WASTE
TYPE, AND BY REGION; TREND ANALYSIS, COMPETITIVE MARKET SHARE \& FORECAST, 2016-26, [online], Available: https://www.researchandmarkets.com/re ports/5024908/mining-wastemanagement-market-by-miningmethod\#rela3-5024805, [Accessed: 21Oct-2021].

[2] A. P. Manuel Caraballo Monge, Robert Ayuso, THE ROLE OF MINE AND INDUSTRIAL WASTES IN THE GROWING DEMAND OF MINERAL RESOURCES, [Online], Available: https://www.sciencedirect.com/journal/j ournal-of-geochemicalexploration/special-issue/1062JK20VCP [Accessed: 21-Oct-2021].

[3] Banco Mundial, LA PRODUCCIÓN MINERA SE DISPARA CON EL AUMENTO DE LA DEMANDA DE ENERGÍA LIMPIA, [Online]. Available:

https://www.bancomundial.org/es/news/ press-release/2020/05/11/mineralproduction-to-soar-as-demand-forclean-energy-increases, [Accessed: 21Oct-2021]. 